\title{
DOUBLE CYSTIC DUCT AS RARE ANATOMIC VARIANT
}

\author{
Antonio Serafin Valero-Liñán, \\ Toni I. Stoyanov, \\ Alba Sanchez- Gallego, \\ Elías Garcia-Grimaldo, \\ José Antonio Gonzalez-Masiá, \\ José Ignacio Miota de Llama, \\ Belén Conde-Inarejos, \\ Olga Luengo-Ballester, \\ Beatriz Aguado-Rodríguez, \\ Kaloyan T. Ivanov ${ }^{1}$, \\ Pencho T. Tonchev²
}

Department of General and Digestive Surgery, Albacete University Hospital Complex, Spain

${ }^{1}$ Department of General and Plastic

Surgery,

University Hospital "Medika",

Ruse,

Bulgaria

2Departments of Surgical

Propaedeutics,

Medical University - Pleven, Bulgaria

\section{Corresponding Author:}

Antonio Serafin Valero-Liñán

Department of General and Digestive

Surgery,

Albacete University Hospital Complex

37, C/ Hermanos Falco Str.

02006, Albacete

Spain

e-mail:aseravl@yahoo.es

Received: April 21, 2019

Revision received: May 20, 2019

Accepted: July 30, 2019

\section{Summary}

The variations of the cystic duct are so common that only $30 \%$ of all humans present the classical anatomical arrangement between the common bile duct (CBD), the cystic duct, and adjacent arteries. Thus, it could be considered that anomalies of the biliary tree are a rule rather than an exception. Duplication of the cystic duct, however, is a very uncommon anatomical finding. In the Department of General and Digestive Surgery of the University Hospital Complex Albacete, a 73-year-old patient was admitted with symptoms of cholangitis. He underwent emergency surgery that found exacerbated chronic cholecystitis and dilation of the CBD. Cholecystectomy was performed with identification of a double cystic duct that drained separately in the CBD and exploration of the last revealed cholangitis without choledocholithiasis. The postoperative period progressed favourably, proceeding to discharge from the hospital with Kehr drainage closed. In conclusion, we consider that the routine use of intraoperative cholangiography when there is suspicion of anatomical variations of the biliary tree is mandatory to rule out lesions or alterations thereof.

Key words: double cystic duct, common bile duct, cholangitis, cholangiography, cholecystectomy

\section{Introduction}

The cystic duct in $66 \%$ of cases has a spiral shape and drains into the common bile duct (CBD) under different angles [1]. The variations of the cystic duct are so common that only a third of all cases show the classical anatomical arrangement between the CBD, the cystic duct, and adjacent arteries [2]. The knowledge of normal anatomy of the biliary tree and its variations is a key to the success of surgical procedures. Duplication of the cystic duct, however, is a very uncommon anatomical finding; there are only 17 cases in the literature thus far $[3,4]$.

\section{The case description}

A 73-year old male with a history of choledocholithiasis, diagnosed by Magnetic Resonance Cholangiography (MRC), was admitted for endoscopic retrograde cholangiopancreatography (ERCP). Family history 
was clear. Comorbidities included diabetes Mellitus and Arterial Hypertension. The patient's physical examination showed deteriorated general condition, hypotension, tachycardia, pain with positive tenderness rebound sign in upper hemiabdomen with prevalence in the right hypochondrium.

After unsuccessful ERCP (identification of choledocholithiasis without extraction of the stones), endoscopic sphincterotomy and placement of the plastic biliary prosthesis in the CBD were done. After the procedure, the patient developed a fever and elevated serum bilirubin levels. At this point, the differential diagnosis included cholangitis with acute perforated cholecystitis (diagnosed by abdominal ultrasound examination), versus post ERCP pancreatitis or post ERCP duodenal perforation.

Emergency surgery was performed, finding exacerbated chronic cholecystitis and CBD dilatation. Open cholecystectomy was performed with identification of double cystic duct that drained separately in the $\mathrm{CBD}$, and its exploration showed cholangitis without verifying choledocholithiasis or plastic biliary prosthesis (Figure 1).

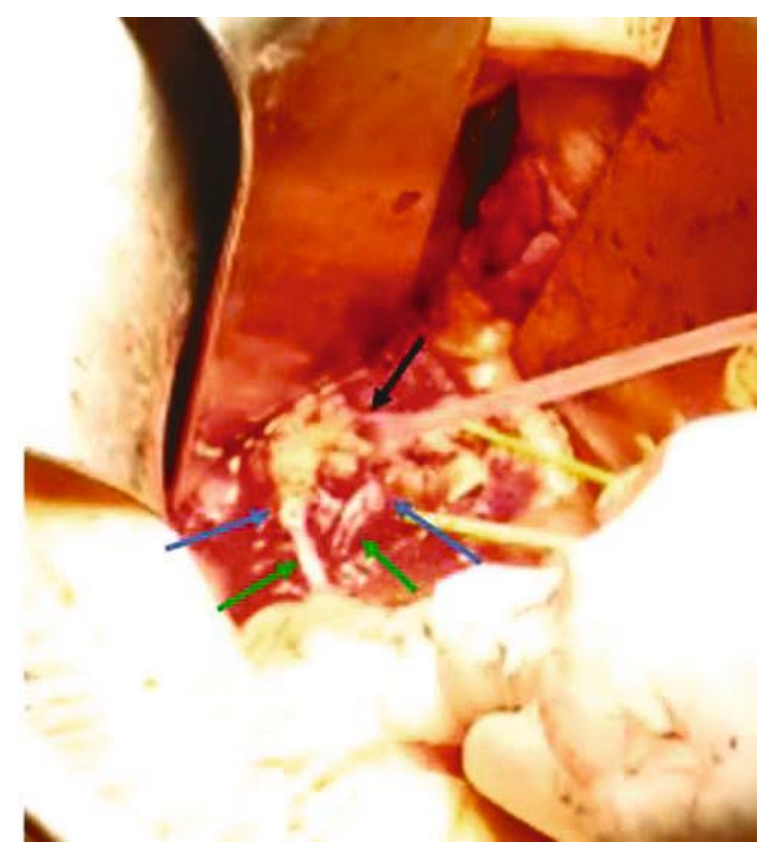

Figure 1. Hepatic hilum after cholecystectomy and CBD exploration. Black arrow: Probe inside the CBD with instillation of saline. Blue arrows: 2 connected cystic ducts. Green arrows: exit of saline by both cystic ducts after instillation through the CBD
Intraoperative cholangiography confirmed double cystic duct, without lesion of CBD or residual choledocholithiasis (Figure 2).

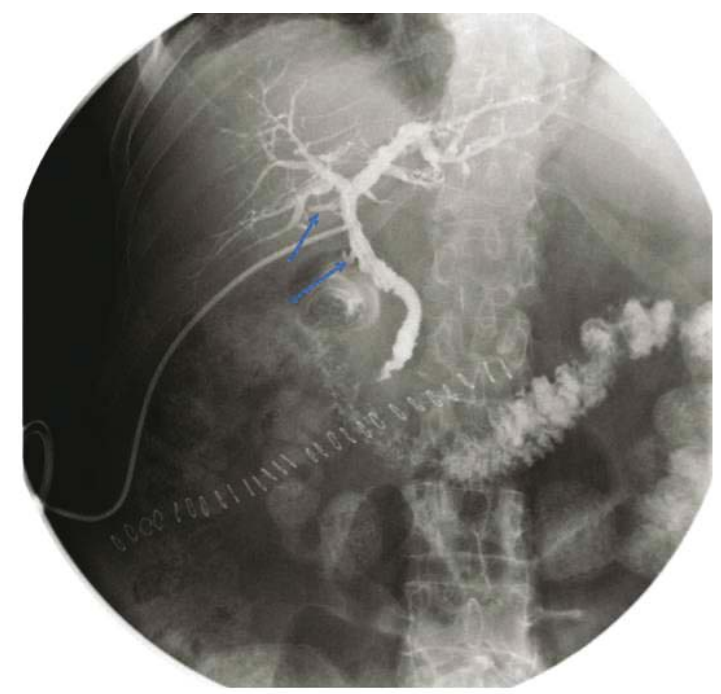

Figure 2. Trans-Kehr intraoperative cholangiography The two cystic ducts can be seen marked with a blue arrow, draining into the $\mathrm{CBD}$, as well as its integrity, the absence of choledocholithiasis and plastic biliary prosthesis, and a good contrast step to the duodenum

Placement of a Kehr drainage and enterotomy with extraction of the plastic biliary prosthesis was performed, after finding it in the proximal jejunum. The postoperative period went favourably. On the seventh postoperative day, trans-Kehr cholangiography was done, showing the normal passage of the contrast to the duodenum without evidence for residual choledocholithiasis. The patient was discharged on the eighth postoperative day with closed Kehr drainage.

Six weeks postoperatively the Kehr drainage was removed in an outpatient setting without incidents. Six months after the operation, the patient is asymptomatic without any analytical alterations in the liver profile.

\section{Discussion}

As we know, the variations of any anatomical structure increase the risks of its injury, the probability of conversion to open surgery, and the possible postoperative complications [5]. Before discussing the double cystic duct, we review the biliary anatomical variations that can give the appearance of this rare anatomical 
variation described by Blumgart et al. (2000) that should be taken into consideration to avoid injury of the bile duct [6]. These are drainage of segment VI towards the cystic duct, drainage of the right posterior sector towards the cystic duct, drainage of the posterior distal or proximal right region of the neck or body of the gallbladder, respectively.

Regarding the double cystic duct, according to the literature, it is usually more common among women $-73 \%$ of cases [4]. Initially, it was thought that the double cystic duct was typically related to double gallbladder $[5,7]$, but according to most recent literature data, this percentage is reduced to $33 \%$ [4]. Its infrequency and difficult ultrasound imaging make preoperative diagnosis very uncommon; thus, the diagnosis is established generally during surgery [1], as in our case. Also, in agreement with other authors $[2,8,9]$, we consider that the routine use of intraoperative cholangiography, when these findings are suspected, is mandatory to prevent bile duct injury or confirm the presence of anatomical variations of the biliary tree.

We strongly believe that knowledge of normal biliary anatomy and that of the most frequent anatomical anomalies is critical for avoiding injuries of the biliary tree. Because of their rarity, these anatomical variants (such as the present case) become a challenge for the surgeon. Therefore, we emphasize the importance of intraoperative cholangiography in these cases. Of course, diagnosis before surgery would be the ideal case [10]. Unfortunately, this rarely happens.

\section{Conclusions}

The double cystic duct is a rare anatomical variant. Its preoperative or intraoperative identification is crucial to prevent lesions of the CBD and to repair these structures if they are damaged intraoperatively. In case of unclear anatomy of the biliary tree, the use of intraoperative cholangiography is mandatory.

\section{Acknowledgements}

The authors declare there are no conflicts of interest and financial sources for this article.

\section{References}

1. Shivhare R, Sikora SS. Double cystic duct: a rare biliary anomaly encountered at laparoscopic cholecystectomy. J LaparoendoscAdv Surg Tech A. 2002;12(5):391-2.

2. Tsutsumi S, Hosouchi Y, ShimuraT, Asao T, Kojima T, Takenoshita SI, et al. Double cystic duct detected by endoscopic retrograde cholangiopancreatography and confirmed by intraoperative cholangiography in laparoscopic cholecystectomy: a case report. Hepatogastroenterology. 2000;47(35):1266-8.

3. Agha RA, Fowler AJ, Saeta A, Barai I, Rajmohan S, Orgill DP, et al. The SCARE Statement: Consensus-based surgical case report guidelines. Int J Surg.2016;34:180-6.

4. Salih AM, Kakamad FH, Mohammed SH, Salih RQ, Habibullah IJ, Muhialdeen AS, et al. Double cystic duct, a review of literature with report of a new case. Int J Surg Case Rep. 2017;38:146-8.

5. Otaibi W, Quach G, Burke B. Double cystic duct in a septated gallbladder. J Investig Med High Impact Case Rep. 2015;3(2):2324709615579105.

6. Blumgart LH, Hann LE. Surgical and radiologic anatomy of the liver and biliary tract, and pancreas. In: Blumgart LH, Fong Y, editors. Surgery of the liver and biliary tract. 3rd ed. London: WB Saunders; 2000. p. 18-20.

7. Görkem SB, Doğanay S, Kahriman G, Küçükaydın M, Coşkun A. Acute cholecystitis of a duplicated gallbladder with double cystic duct in a 10 year old boy. Balkan Med J. 2014;31(4):366-7.

8. Samnani SS, Ali A. "Y" variant of double cystic duct: incidental finding during laparoscopic cholecystectomy. Indian J Surg. 2015;77(Suppl 3):S1491.

9. Munie S, Nasser H, Go PH, Rosso K, Woodward A. Case report of a duplicated cystic duct: a unique challenge for the laparoscopic surgeon. Int J Surg Case Rep. 2019;56:78-81.

10. Fujii A, Hiraki M, Egawa N, Kono H, Ide T, Nojiri J, et al. Double cystic duct preoperatively diagnosed and successfully treated with laparoscopic cholecystectomy: a case report. Int J Surg Case Rep. 2017;37:102-5. 